\title{
Infolding and collapse of thoracic endoprostheses: Manifestations and treatment options
}

\author{
Daniel Y. Sze, MD, PhD, ${ }^{a}$ R. Scott Mitchell, MD, ${ }^{b}$ D. Craig Miller, MD, ${ }^{b}$ Dominik Fleischmann, MD, ${ }^{c}$ \\ Joan K. Frisoli, MD, PhD, ${ }^{\text {a }}$ Stephen T. Kee, MD, ${ }^{a}$ Archana Verma, MD, ${ }^{a}$ Michael P. Sheehan, MSN, RN, \\ FNP, ${ }^{b}$ and Michael D. Dake, MD ${ }^{\mathrm{a}, \mathrm{b}}$
}

Objectives: We sought to review the clinical sequelae and imaging manifestations of thoracic aortic endograft collapses and infoldings and to evaluate minimally invasive methods of repairing such collapses.

\begin{abstract}
Methods: Two hundred twenty-one Gore endografts (Excluder, TAG; W. L. Gore \& Associates, Inc, Flagstaff, Ariz) were deployed in 145 patients for treatment of pathologies including aneurysms and pseudoaneurysms, dissections, penetrating ulcers, transections, fistulae, mycotic aneurysms, and neoplastic invasions in 6 different prospective trials at a single institution from 1997 to 2007. Device collapses and infoldings were analyzed retrospectively, including review of anatomic parameters, pathologies treated, device sizing and selection, clinical sequelae, methods of repair, and outcome.

Results: Six device collapses and infoldings were identified. Oversized devices placed into small-diameter aortas and imperfect proximal apposition to the lesser curvature were seen in all proximal collapses, affecting patients with transections and pseudoaneurysms. Infoldings in patients undergoing dissection represented incomplete initial expansion rather than delayed collapse. Delayed collapse occurred as many as 6 years after initial successful deployment, apparently as a result of changes in the aortic configuration from aneurysmal shrinkage. Clinical manifestations ranged from life-threatening ischemia to complete lack of symptoms. Collapses requiring therapy were remedied percutaneously by bare stenting or in one case by branch vessel embolization.
\end{abstract}

Conclusions: Use of oversized devices in small aortas carries a risk of device failure by collapse, which can occur immediately or after years of delay. When clinically indicated, percutaneous repair can be effectively performed.

Since its original introduction into clinical trials in 1997 as the Thoracic Excluder and its subsequent redesign in 2003 as the TAG, the Gore thoracic endograft device (W. L. Gore \& Associates, Inc, Flagstaff, Ariz) has received CE Marking and US Food and Drug Administration (FDA) Premarket Approval for treatment of aneurysms of the descending thoracic aorta, and thousands of devices have been implanted worldwide. Indications and anatomic inclusion criteria are explicitly specified, ${ }^{1}$ but numerous trials and off-label uses of the devices have pushed beyond these established boundaries. Applications in situations outside of the physical conditions for which the device was designed and bench tested could result in device failure. Several case reports have described partial or total collapse of TAG devices, all leading to further major intervention. ${ }^{2-12}$ We describe our experience with 6 recognized device collapses and infoldings, review the potential causative factors

From the Division of Interventional Radiology, ${ }^{a}$ the Department of Cardiothoracic Surgery, ${ }^{\mathrm{b}}$ and the Department of Radiology, ${ }^{\mathrm{c}}$ Stanford University, Stanford, Calif. Received for publication Sept 4, 2008; revisions received Nov 6, 2008; accepted for publication Dec 14, 2008.

Reprint requests: Daniel Y. Sze, MD, PhD, H-3646 Stanford University Medical Center, 300 Pasteur Dr, Stanford, CA 94305-5642 (E-mail: dansze@stanford.edu).

J Thorac Cardiovasc Surg 2009;138:324-33

$0022-5223 / \$ 36.00$

Copyright (c) 2009 by The American Association for Thoracic Surgery doi: 10.1016/j.jtcvs.2008.12.007 leading to collapse, and illustrate several different conservative and minimally invasive treatment options.

\section{MATERIALS AND METHODS}

All patient data were handled in accordance to Health Insurance Portability and Accountability Act regulations. All 6 protocols in which patients were enrolled were approved by the institutional review board.

From 1997 to July 2004, 136 Gore Thoracic Excluder devices were implanted into 87 patients at a single academic institution under 2 different Gore-sponsored FDA trials and 1 Investigator-sponsored Investigational Device Exemption (ISIDE) trial. From November 2003 to October 2007, an additional 85 TAG devices were implanted into 58 patients in 3 different Gore-sponsored trials and the continuation of the ISIDE. Device-related adverse events were prospectively documented in the mandatory follow-up protocols and retrospectively reviewed for this study.

\section{RESULTS}

Six device collapses and infoldings were documented, including 3 acute collapses in patients treated for traumatic injury of the aorta, 2 infoldings in patients with acute and subacute aortic dissection, and 1 delayed collapse in a patient treated for an anastomotic pseudoaneurysm (Table 1). The delayed collapse involved a Thoracic Excluder more than 6 years after original deployment, and the other 5 involved TAG devices.

\section{Patient 1}

A 23-year-old man presented to an outside hospital with a complex bullet injury after an apparent attempted assault 


\author{
Abbreviations and Acronyms \\ CT = computed tomographic \\ FDA $=$ US Food and Drug Administration \\ IFU = Instructions for Use \\ ISIDE $=$ Investigator-sponsored Investigational \\ Device Exemption \\ LSCA = left subclavian artery \\ PIT = primary intimal tear
}

on his cervical spinal cord 1 day after his being released from incarceration. The bullet transgressed his mastoid region, mandible, and hyoid and lodged in his left thorax. Because of a VI/VI bruit in his thorax, a computed tomographic (CT) scan was performed, which showed a fistula from his descending thoracic aorta extending cranially through the mediastinum and into the left brachiocephalic vein, which had been transgressed by the bullet. He was electively intubated and transferred to our institution for treatment.

The patient was enrolled into the ISIDE. The proximal and distal landing areas measured 21 and $19 \mathrm{~mm}$ in average diameter, respectively. A $26 \mathrm{~mm} \times 10 \mathrm{~cm}$ TAG was deployed over the aortic injury, intentionally covering the origin of the left subclavian artery (LSCA). Postdeployment plasty was performed by using a compliant trilobed balloon (Gore), and the proximal end of the device appeared well apposed to the aortic wall without a substantial "bird beak", (Table 2). ${ }^{13}$

The bruit resolved but returned to III/VI severity 3 days later. A chest radiograph showed distortion of the endograft, and a CT angiogram confirmed partial collapse of the proximal $90 \%$ with a C-shaped cross-section, which was concave toward the aortic lesser curve (Figure 1). At angiographic reintervention, the TAG was easily re-expanded with an angioplasty balloon but collapsed again immediately on deflation of the balloon. Two balloon-expandable stents (Palmaz XL 3110, Genesis XD 3910; Cordis/J\&J, Warren, NJ) were deployed within the proximal and mid endograft, respectively, and dilated to $22 \mathrm{~mm}$ in diameter. Follow-up radiographs and CT angiograms showed patency of the endograft and elimination of the fistula. The patient was discharged 5 days after bare stent placement but was quickly lost to follow-up (Table 3).

\section{Patient 2}

A 44-year-old man experienced polytrauma from a motorcycle accident, including subarachnoid hemorrhage, grade 3 splenic laceration, displaced femoral fracture, and multiple rib fractures. The proximal and distal landing areas measured 24 and $20 \mathrm{~mm}$ in average diameter, respectively. $\mathrm{He}$ was enrolled in the ISIDE, and a $26 \mathrm{~mm} \times 10 \mathrm{~cm}$ TAG was deployed over a Gavant grade 3 transection at the isthmus. ${ }^{14}$ An 18-mm length of the proximal device did not appose the aortic wall in the lesser curvature, despite repeated balloon plasty.

A follow-up chest radiograph on postprocedure day 3 showed collapse, but by the time the radiograph was interpreted, the patient had undergone general anesthesia for orthopedic fixation. Intraoperatively, the patient became anuric with a creatinine value of $2.1 \mathrm{mg} / \mathrm{dL}$, acidotic with a $\mathrm{pH}$ of 7.24 and a serum bicarbonate value of $18 \mathrm{mEq} / \mathrm{L}$, all new abnormalities since the previous day. Immediately after completion of orthopedic fixation, the patient underwent angiographic analysis, which revealed a $30 \mathrm{~mm} \mathrm{Hg}$ mean gradient across the collapsed endograft. The collapse was repaired with a Palmaz 3110 stent dilated to $22 \mathrm{~mm}$ in diameter. A small thrombus was evidently extruded into the left subclavian artery by the stent deployment, requiring thrombectomy with the AngioJet device (Possis, Minneapolis, Minn). No evidence of stroke or left upper extremity ischemia was detected. Metabolic parameters normalized within

TABLE 1. Patient demographics

\begin{tabular}{|c|c|c|c|c|}
\hline $\begin{array}{l}\text { Patient } \\
\text { no. }\end{array}$ & $\begin{array}{l}\text { Age at time of } \\
\text { treatment } \\
(y)\end{array}$ & Sex & Aortic pathology & Comorbidities \\
\hline 1 & 23 & M & $\begin{array}{l}\text { Traumatic arteriovenous } \\
\text { fistula from gunshot wound }\end{array}$ & Mandibular and hyoid fractures, respiratory failure \\
\hline 2 & 44 & M & $\begin{array}{l}\text { Traumatic transection, motorcycle } \\
\text { accident }\end{array}$ & Subarachnoid hemorrhage, splenic laceration, fractures of femur and ribs \\
\hline 3 & 40 & M & $\begin{array}{l}\text { Traumatic transection, motor vehicle } \\
\text { accident }\end{array}$ & $\begin{array}{l}\text { Atlanto-occipital dislocation; epidural, subdural, subarachnoid, } \\
\text { and intraventricular hemorrhage; renal contusion; pulmonary } \\
\text { embolism; fractures of pelvis, scapula, and } 11 \text { ribs }\end{array}$ \\
\hline 4 & 19 & M & Acute type B dissection & $\begin{array}{l}\text { End-stage renal disease, hypertension, transplant nephrectomy } \\
\text { for chronic rejection }\end{array}$ \\
\hline 5 & 42 & M & Subacute type B dissection & Hypertension, diabetes, acute renal failure \\
\hline 6 & 67 & M & Anastomotic pseudoaneurysm & $\begin{array}{l}\text { Prior thoracotomies } \times 2 \text { for repair of coarctation, history of rheumatic } \\
\text { fever with recurrent endocarditis }\end{array}$ \\
\hline
\end{tabular}


TABLE 2. Anatomy and device-related parameters

\begin{tabular}{|c|c|c|c|c|c|c|}
\hline $\begin{array}{c}\text { Patient } \\
\text { no. }\end{array}$ & $\begin{array}{c}\text { Devices used } \\
\text { (mm diameter } \times \\
\text { cm length) }\end{array}$ & $\begin{array}{c}\text { Proximal } \\
\text { landing zone } \\
\text { diameters } \\
\text { (at } 2,1 \text {, and } \\
0 \mathrm{~cm} \text { above } \\
\text { lesion; mm)* }\end{array}$ & $\begin{array}{c}\text { Distal } \\
\text { landing zone } \\
\text { diameters } \\
\text { (at } 0,1 \text {, and } \\
2 \mathrm{~cm} \text { below } \\
\text { lesion; } \mathrm{mm} \text { )* }\end{array}$ & $\begin{array}{c}\text { Minimum } \\
\text { diameter in } \\
\text { treated segment } \\
\text { after } \\
\text { deployment } \\
(\mathbf{m m})^{*} \\
\end{array}$ & $\begin{array}{c}\text { Balloon } \\
\text { plasty } \\
\text { performed } \\
\text { after } \\
\text { initial } \\
\text { placement }\end{array}$ & $\begin{array}{c}\text { Length of lack } \\
\text { of apposition } \\
\text { of device to } \\
\text { proximal lesser } \\
\text { curvature (bird beak), } \\
\text { including flares (mm) }\end{array}$ \\
\hline 1 & $26 \times 10 \mathrm{TAG}$ & $21,20,17$ & $19,19,19$ & 19 & Yes & 10 \\
\hline 2 & $26 \times 10 \mathrm{TAG}$ & $25,24,25$ & $20,20,21$ & 22 & Yes & 18 \\
\hline 3 & $26 \times 10 \mathrm{TAG}$ & $24,23,23$ & $23,24,22$ & 23 & No & 5 \\
\hline 4 & $26 \times 10 \mathrm{TAG}$ & $23,22,21$ & $21,20,18$ & $21 \times 11(16)$ & No & 0 \\
\hline 5 & $34 \times 15$ TAG & $29,29,32$ & $\begin{array}{l}25 \times 7(17) \\
23 \times 7(16) \\
18 \times 10(14)\end{array}$ & $21 \times 16(19)$ & No & 21 \\
\hline 6 & $\begin{aligned} & 26 \times 12.5, \\
& 26 \times 7.5 \\
& 31 \times 15 \\
& 26 \times 7.5 \\
& \text { Thoracic excluders }\end{aligned}$ & $\begin{array}{l}32 \times 26(29) \\
28 \times 24(26) \\
26 \times 22(24)\end{array}$ & $26,26,26$ & $24 \times 18(21)$ & Yes & 21 \\
\hline
\end{tabular}

*Where luminal cross-sections were not circular, they were assumed to be elliptical. Major and minor axes were measured, perimeter was calculated by using the method of Ramanujan, ${ }^{13}$ and the approximate equivalent circular diameter was then calculated (shown in parentheses).

2 days, and the patient has had no further aortic issues in 22 months of follow-up.

\section{Patient 3}

A 40-year-old man experienced polytrauma from a motor vehicle accident, including atlanto-occipital dislocation; subarachnoid, subdural, epidural, and intraventricular hemorrhage; renal contusion; pulmonary embolism; and fractures of the pelvis, scapula, and 11 ribs. He was enrolled in the ISIDE, and a $26 \mathrm{~mm} \times 10 \mathrm{~cm}$ TAG was deployed over his grade 3 aortic transection at the isthmus. The proximal and distal landing areas measured 23 and $23 \mathrm{~mm}$ in average diameter, respectively. A 12-mm length of the proximal device did not appose the aortic wall in the lesser curvature but was not subjected to balloon plasty. Collapse was diagnosed by means of chest radiography on postprocedure day 3 , but the patient was still so acutely ill from associated injuries that no suspicion was raised for collapse based on clinical signs. The patient was brought to angiographic reintervention on an emergency basis, where a 35 $\mathrm{mm} \mathrm{Hg}$ mean gradient was measured. The proximal and mid TAG were reinforced with 2 Palmaz 3110 stents dilated to 25 and $23 \mathrm{~mm}$ in diameter, respectively. This patient has been free of aortic issues in 31 months of follow-up.

\section{Patient 4}

A 19-year-old man with chronic hypertension presented with acute chest and back pain and hypotension during hemodialysis. He had previously undergone transplant nephrectomy for chronic rejection caused by noncompliance with immunosuppressive medications. CT scanning of the chest revealed an acute type B dissection, with the primary intimal tear (PIT) located $7 \mathrm{~cm}$ distal to the origin of the left subclavian artery. The proximal and distal landing areas measured 22 and $18 \mathrm{~mm}$ in average diameter, respectively, with a minimal true-lumen diameter of $17 \mathrm{~mm}$ in between. He was enrolled in the ISIDE, with consent provided by his guardians. A $26 \mathrm{~mm} \times 10 \mathrm{~cm}$ TAG was deployed over the PIT, but the device landed approximately $20 \mathrm{~mm}$ distal to the measured landing area, and the proximal end of the device landed in an area where the true lumen measured $17 \mathrm{~mm}$ in diameter before device deployment. Balloon plasty was avoided because of the presumed fragility of the dissection flap.

Infolding of the proximal half of the endograft was demonstrated by means of CT angiographic imaging 3 days later, along with a moderate type I proximal fixation endoleak, allowing continued opacification of the false lumen. In retrospect, the procedural fluoroscopic images suggested that the proximal portion of the device never fully opened, constrained by the dissection flap. The patient's family refused to consent to additional treatment but partially complied with the follow-up protocol. The endoleak resolved spontaneously within 6 months, and the false lumen thrombosed and regressed completely, despite persistent infolding of the proximal half of the endograft. In 17 months of follow-up, the patient demonstrated no evidence of distal ischemic or embolic complications but died from complications of end-stage renal failure.

\section{Patient 5}

A 42-year-old man with a history of hypertension presented twice to an outside hospital with abdominal and flank pain but was discharged after brief and unremarkable 

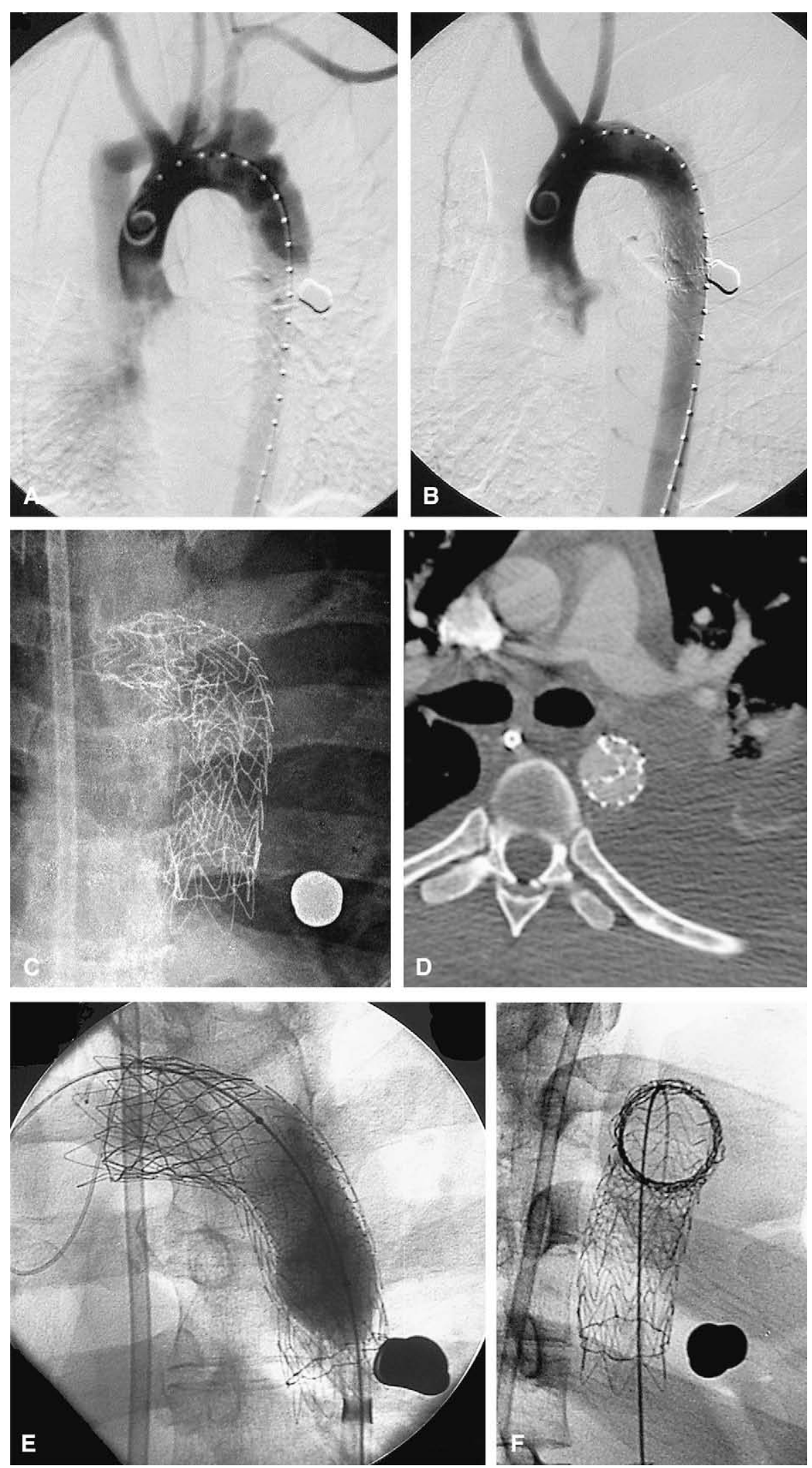

FIGURE 1. A, Thoracic aortogram of a 23-year-old man with a gunshot wound that caused a large arteriovenous fistula extending from the proximal descending thoracic aorta into the left brachiocephalic vein. B, The fistula was closed by means of placement of a $26 \mathrm{~mm} \times 10 \mathrm{~cm}$ TAG over the aortic injury 
TABLE 3. Characterization of collapses, treatments, and clinical outcomes

\begin{tabular}{|c|c|c|c|c|c|c|c|c|}
\hline $\begin{array}{c}\text { Patient } \\
\text { no. }\end{array}$ & $\begin{array}{c}\text { Interval } \\
\text { between initial } \\
\text { treatment and } \\
\text { collapse }\end{array}$ & $\begin{array}{c}\text { Portion of } \\
\text { device } \\
\text { collapsed }\end{array}$ & $\begin{array}{c}\text { Length } \\
\text { of collapse }(\mathbf{c m})\end{array}$ & $\begin{array}{l}\text { Direction } \\
\text { of concavity } \\
\text { of collapse }\end{array}$ & $\begin{array}{c}\text { Minimum } \\
\text { luminal } \\
\text { diameter(mm) }\end{array}$ & $\begin{array}{l}\text { Clinical } \\
\text { signs and } \\
\text { symptoms }\end{array}$ & $\begin{array}{l}\text { Secondary } \\
\text { procedures }\end{array}$ & Outcome \\
\hline 1 & $3 \mathrm{~d}$ & Proximal/mid & 9 & $\begin{array}{c}\text { Toward lesser } \\
\text { curvature }\end{array}$ & $25 \times 15(20)$ & None & Bare stent $\times 2$ & Resolved \\
\hline 2 & $3 \mathrm{~d}$ & Proximal/mid & 8 & $\begin{array}{c}\text { Toward lesser } \\
\text { curvature }\end{array}$ & 9 & $\begin{array}{l}\text { Anuria, } \\
\text { acidemia; } \\
30 \mathrm{~mm} \mathrm{Hg} \\
\text { gradient }\end{array}$ & $\begin{array}{l}\text { Bare stent, } \\
\quad \text { subclavian } \\
\text { thrombectomy }\end{array}$ & Resolved \\
\hline 3 & $3 \mathrm{~d}$ & Proximal/mid & 8 & $\begin{array}{c}\text { Toward lesser } \\
\text { curvature }\end{array}$ & 11 & $\begin{array}{l}\text { None; } 35 \\
\text { mm Hg } \\
\text { gradient }\end{array}$ & Bare stent & Resolved \\
\hline 4 & $3 \mathrm{~d}$ & Proximal & 5 & $\begin{array}{l}\text { Toward true lumen } \\
\text { (lesser } \\
\text { curvature) }\end{array}$ & $21 \times 11(16)$ & None & None & $\begin{array}{c}\text { Type } 1 \text { leak } \\
\text { resolved, } \\
\text { device } \\
\text { remained } \\
\text { collapsed }\end{array}$ \\
\hline 5 & $4 \mathrm{~d}$ & Mid/distal & 9 & $\begin{array}{l}\text { Toward false } \\
\text { lumen (greater } \\
\text { curvature) }\end{array}$ & $21 \times 16(19)$ & $\begin{array}{l}\text { None; no } \\
\text { pressure } \\
\text { gradient }\end{array}$ & $\begin{array}{l}\text { Coil } \\
\text { embolization } \\
\text { of left } \\
\text { subclavian } \\
\text { artery and } \\
\text { right T8 } \\
\text { intercostal } \\
\text { artery }\end{array}$ & $\begin{array}{l}\text { Leak resolved, } \\
\text { stent graft } \\
\text { gradually } \\
\text { opened but } \\
\text { eroded through } \\
\text { flap }\end{array}$ \\
\hline 6 & $6.5 \mathrm{y}$ & Entire & 7.5 & $\begin{array}{c}\text { Toward lesser } \\
\text { curvature }\end{array}$ & 0 & None & None & $\begin{array}{l}\text { Stable but } \\
\text { collapsed }\end{array}$ \\
\hline
\end{tabular}

examinations. On his third visit to the hospital, a CT scan was performed, showing a type B aortic dissection with poor perfusion of the left kidney. He was intubated for respiratory failure and transferred to our institution for care. The intact aorta proximal to the dissection measured $31 \mathrm{~mm}$ in diameter, but the true lumen was attenuated to an $18 \times 10$ $\mathrm{mm}$ crescent in the midthorax. He was enrolled in the ISIDE, and a $34 \mathrm{~mm} \times 15 \mathrm{~cm}$ TAG was deployed distal to the origin of the left common carotid artery, covering the PIT, which extended into the origin of the intentionally covered LSCA. The distal half of the endograft did not fully expand after deployment, but balloon dilatation was avoided because of the risk of causing a secondary intimal tear. Angiography revealed restored flow to the mesenteric branches, and a bare stent was placed in the dissected left renal artery to obliterate the nonreentrant false lumen.

A CT angiogram 4 days after the procedure revealed persistent infolding of the distal half of the endograft, which was concave toward the false lumen. In addition, there was persistent early contrast opacification of the aortic false lumen, as well as of the covered LSCA. Because the symptoms of distal ischemia had resolved, no reintervention was performed before discharge.

In follow-up, the endoleak persisted, resulting in aneurysmal dilatation of the false lumen and an increase in total aortic diameter from 38 to $52 \mathrm{~mm}$ within 9 months. At 11 months, the origin of the LSCA was coil embolized, with coils extending from the true lumen of the LSCA across the PIT into the false lumen of the aorta (Figure 2). This resulted in thrombosis of the false lumen proximally but with persistent filling distally through natural fenestrations and drainage into bronchial and intercostal arteries. Because of progressive false lumen enlargement in the patent segment at 19 months, the largest of the intercostal arteries was coil embolized, eventually resulting in complete thrombosis of the thoracic false lumen, enlargement of the true lumen,

covering the left subclavian artery. The covered portion of the aorta tapered from 21 to $19 \mathrm{~mm}$ in diameter. C, An anteroposterior chest radiograph on postoperative day 3 showed distortion of the proximal aspect of the device. D, Axial image from a contrast medium-enhanced computed tomographic scan confirmed collapse of the stent graft. E, Real-time fluoroscopic imaging revealed dynamic collapse varying in severity according to the cardiac cycle. The collapse was repaired by using 2 balloon-expandable stents in the proximal and mid portions of the stent graft. F, Right anterior oblique spot image of the repaired device showed restoration of the circular cross-section. Note the residual redundancy of the distal gold ring, which is indicative of incomplete expansion from constraint within an undersized vessel. 

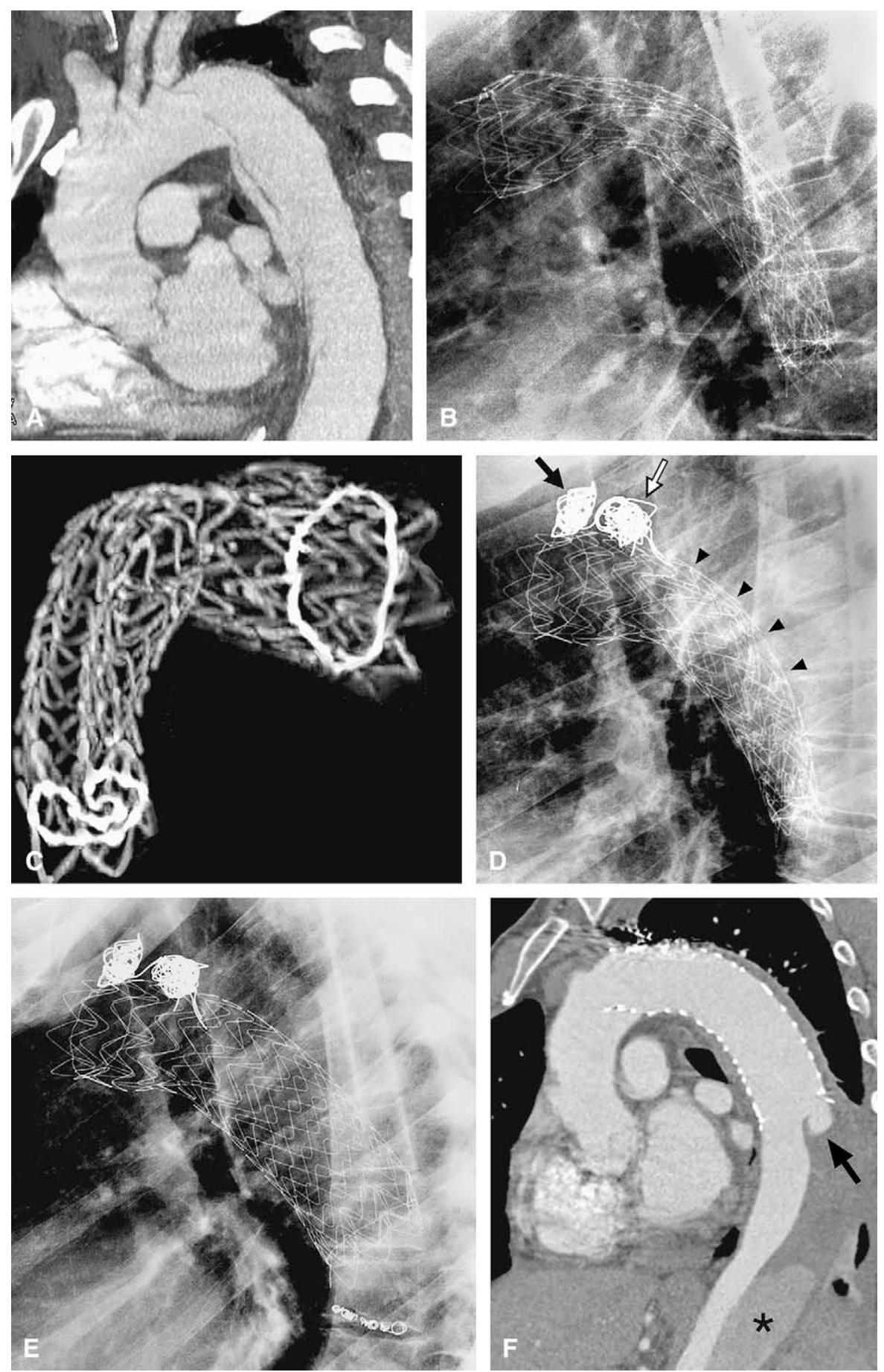

FIGURE 2. A, Curved planar reconstruction of a computed tomographic angiogram performed on a 42 -year-old man 20 days after onset of back, flank, and abdominal pain; anorexia; and increased hypertension showed a type B dissection with nearly complete obliteration of the aortic true lumen. The undissected arch measured $31 \mathrm{~mm}$ in diameter. The dissection flap extended into the left subclavian artery. B, Lateral chest radiograph 3 days after deployment of a $34 \mathrm{~mm}$ $\times 15 \mathrm{~cm}$ TAG over the primary intimal tear and covering the left subclavian artery showed incomplete expansion of the distal portion of the device, which was not recognized at the time of deployment. C, Posterior view maximum-intensity projection of the postprocedural computed tomographic angiogram demonstrated the seam of infolding extending along the distal $9 \mathrm{~cm}$ with a crescentic cross-section at the level of the distal gold band. A complex endoleak was observed involving the proximal landing area and the left subclavian artery. D, Lateral chest radiograph 3 months after coil embolization of the origin of the left subclavian artery showed coils extending into the false lumen of the aorta (hollow arrow) across the portion of the primary intimal tear extending into the left subclavian artery (solid arrow), the source of a complex endoleak. The midportion of the device showed interval expansion caused by the thrombosis and relief of pressurization of the false lumen in the proximal descending thoracic aorta (arrowheads), but the distal infolding remained. E, Lateral chest 

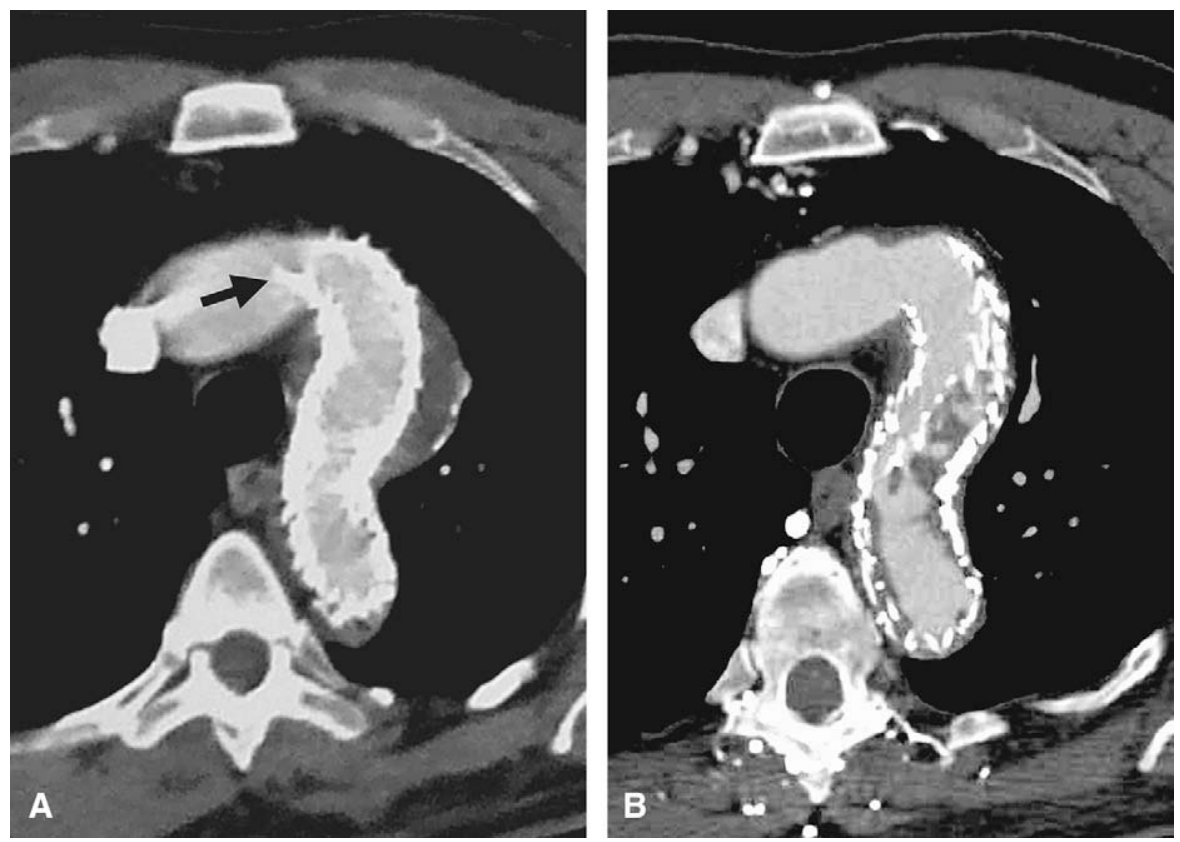

FIGURE 3. A, Curved planar reconstruction of a computed tomographic angiogram performed on a 67-year-old man after stent graft repair of an anastomotic pseudoaneurysm 47 years after open repair of an aortic coarctation showed incomplete apposition of the device to the medial lesser curvature of the aorta at the level of a kink. The medial aspect of the stent graft protruded into the arch by $21 \mathrm{~mm}$ (arrow), even though the lateral aspect was well apposed because of a sharp angulation in the aorta. An intraoperative type Ia endoleak necessitated 2 proximal extensions, successfully excluding the pseudoaneurysm but resulting in this "bird beak." B, Follow-up computed tomographic angiography imaging 6 years later showed collapse of the most proximal stent graft, with thrombus accumulated within the collapsed lumen, blood flow external to the collapsed device, and complete thrombosis and resorption of the pseudoaneurysm. The patient remains asymptomatic and has declined reintervention.

and spontaneous unfolding of the collapsed endograft at 21 months. However, an ulcer formed at the distal end of the stent graft, evidently from erosion through the flap into the false lumen. At 31 months, the patient presented with mild hemoptysis believed to be due to left upper lobe adhesion to the aorta. The patient underwent open surgical partial explantation, graft repair, and lysis of adhesions to the lung but died on postoperative day 4 from complications of postoperative ventricular fibrillation arrest.

\section{Patient 6}

A 67-year-old asymptomatic man was given a diagnosis of an enlarging pseudoaneurysm by means of echocardiographic imaging after having undergone surgical repair of aortic coarctation and ventricular septal defect at age 20 years. He was enrolled in the 03-03 Pivotal Gore trial, although with a protocol deviation because of his prior operation. The distal aortic arch was severely kinked, with a diameter at the kink of $24 \times 18 \mathrm{~mm}$. The 2 Thoracic Excluder devices originally deployed did not create an adequate proximal seal. Two successive extensions were deployed to achieve a better proximal seal. The most proximal of these, a $26 \mathrm{~mm} \times 7.5 \mathrm{~cm}$ device, jutted into the kink, where the proximal device was constrained to a diameter of $24 \times 18 \mathrm{~mm}$. In addition, a 21-mm length of the proximal device did not appose the lesser curvature (Figure 3).

The sac was successfully excluded, and the patient remained asymptomatic as the pseudoaneurysm diminished in size. A follow-up CT scan performed 79 months after endograft repair showed complete collapse of the proximal device with well-maintained distal perfusion. A moderate amount of thrombus was detected within the residual lumen of the collapsed device. Because of a lack of symptoms, the patient did not wish to undergo further procedures but underwent follow-up CT scans 13 and 21 months later, which showed no change. The aortic diameter has decreased from 79 to $39 \mathrm{~mm}$, and the aneurysmal sac has decreased from 56 to $9 \mathrm{~mm}$ in diameter. Resting ankle brachial indices were 0.8 bilaterally, with no significant change after exercise. With continued lack of symptoms and a high predicted risk of distal embolization with manipulation of the collapsed stent, no further interventions have been performed.

radiograph taken 3 months after coil embolization of the right T8 intercostal artery showed complete expansion of the entire stent graft. F, Computed tomographic angiographic imaging also showed complete thrombosis and regression of the false lumen at the level of the stent graft, although with a focal erosion through the intimal flap causing an ulcer-like excavation into the false lumen at the distal end of the stent graft (arrow). The infradiaphragmatic false lumen remained patent from flow through natural fenestrations (asterisk). 


\section{DISCUSSION}

Since the introduction of the redesigned Gore thoracic endoprosthesis in 2003, the subtle engineering modifications have manifested as equally subtle changes in clinical performance. ${ }^{15}$ Although the observation of longitudinal spine fractures, as was observed in the preceding Thoracic Excluder device, was completely eliminated by elimination of the spine altogether, the newer TAG device appears susceptible to other mechanical events, including partial or total collapse. Recognition of this potentially catastrophic event has heightened the awareness of the specific guidelines in the Instructions for Use (IFU) ${ }^{1}$ and has spread a message of caution to those interested in potential off-label application of the device. All 6 of the collapses described here involved patients enrolled in protocols addressing indications other than aneurysms, the indication for which the device is currently approved.

At least 22 incidents of TAG collapse have appeared in the literature (Table 4), ${ }^{2-12}$ most of which occurred in patients treated for blunt traumatic injury. The emerging consensus in the literature is that the major risk for collapse is use in a small-diameter aorta outside of the recommendations specified in the IFU. Steinbauer and colleagues ${ }^{11}$ refuted this but in fact misquoted the IFU as allowing "oversizing of $25 \%$," which is inaccurate for the smallest-diameter devices ( 26 and $28 \mathrm{~mm}$ diameter), which allow for only $13 \%$ and $16 \%$ oversizing. Muhs and associates ${ }^{8}$ found that minimum aortic diameter and distal landing zone diameter were the only 2 variables that predicted collapse. Other suspected factors, including arch curvature and length of lack of apposition, did not reach statistical significance, although small sample size limited conclusions. One of our patients, even in retrospect, had aortic dimensions just within the IFU specifications but had incomplete apposition proximally in the acutely curved distal arch, suggesting some contribution from difficult geometry and imperfect apposition. Regardless of the pathology being treated, these data highlight the importance of heeding the anatomic inclusion criteria specified by the manufacturer.

Gore's own comprehensive system of reporting has identified a total of 95 device compressions resulting in 2 devicerelated deaths of a total of 25,000 devices distributed worldwide. ${ }^{16}$ Each of these identified cases has undergone retrospective failure analysis, including meticulous remeasurement of aortic anatomic dimensions by using 3-dimensional reconstructions. They concluded that the root cause of compression is multifactorial, with the highest reported incidence in young patients presenting with acute traumatic transection. These patients generally had aortic diameters of less than 23 $\mathrm{mm}$, had tight radii of curvature of the aortic arch, and would be expected to have peak blood flow velocities and aortic wall elastic pulsatility values significantly greater than in older patients with degenerative, atherosclerotic aneurysmal disease.
In addition, the TAG device was originally designed and subsequently approved by the FDA to treat aneurysms, where the intervening aorta between the 2 landing zones is likely to be larger in diameter than either landing zone. This anatomic configuration is unlikely to be true in the cases of blunt traumatic transections and aortic dissections, the pathologies most frequently associated with endograft collapse. Additional measurements of aortic dimensions along the entire length of the endografted aorta, as well as estimation of the compliance of the disrupted wall or dissection flap, might need to be factored into procedural planning in these patient populations. In fact, the 2 "collapses" in our patients undergoing dissection were actually incomplete expansions from the time of deployment and not delayed collapses of fully expanded devices.

Controversy still remains about how best to treat smallerdiameter aortas, many of which are afflicted by pathologies other than degenerative aneurysms. For patients in whom conventional open surgical intervention is prohibitively risky, other endovascular options can be considered, although data are limited. Options include using endografts of smaller diameter, such as abdominal aortic or iliac components, or the Medtronic Talent Thoracic device (Medtronic, Minneapolis, Minn), which is available to treat aortas as small as $22 \mathrm{~mm}$. Alternatively, radial hoop strength can be increased by using devices from other manufacturers (Talent and Zenith TX2 [Cook Medical, Inc, Bloomington, Ind], available down to $28 \mathrm{~mm}$ in diameter), by prophylactically placing balloon-expandable stents, or by prophylactically deploying 2 superimposed TAG devices.

The clinical complications resulting from collapse vary widely in the literature, from recurrent aneurysm perfusion and distal ischemia caused by aortic occlusion to complete lack of symptoms. Our series of patients similarly presented with signs and symptoms ranging from the life-threatening to the unnoticeable. In addition, 2 of our collapses occurred in the perioperative period in severely injured patients, in whom more subtle signs and symptoms of endograft collapse might have been masked amid the more dramatic sequelae of the original injury. One of our patients (and perhaps one in the series published by Muhs and associates ${ }^{8}$ ) has a collapse so severe that no symptoms are evident and adequate perfusion of distal beds appears to be through an effective "false lumen" outside of the collapsed endograft. Thus, collapse is not necessarily catastrophic and might not even warrant correction in some patients.

When warranted, repair of collapse can be accomplished by means of any of a variety of percutaneous or surgical methods. Several reported patients underwent explantation and surgical repair, which has the benefits of known durability and impossibility of recurrent collapse. Thoracotomy, even in a patient who has recovered from an initial aortic catastrophe, still carries substantial morbidity and mortality. Especially in the 


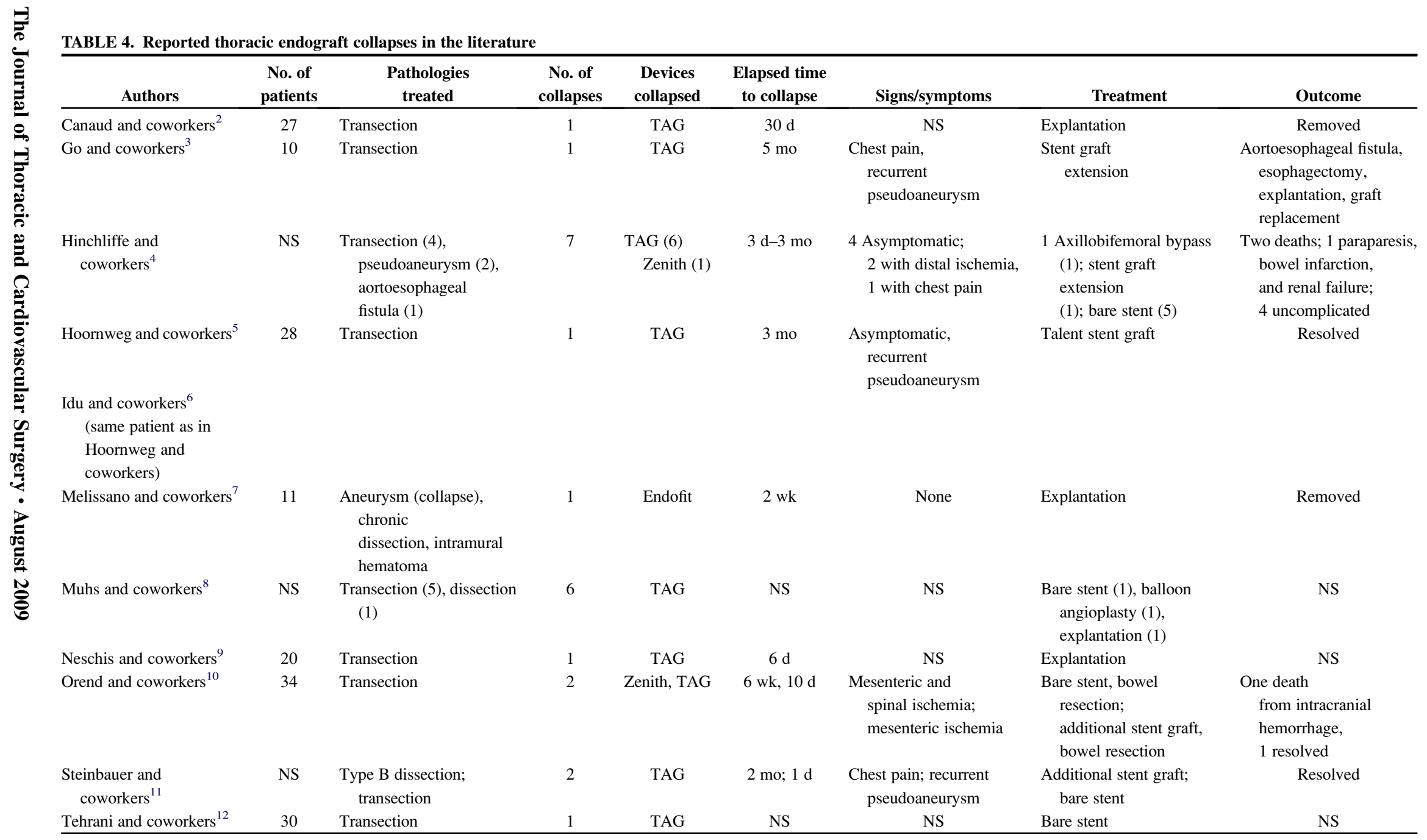


patient who has not yet recovered from concurrent injuries or comorbidities, percutaneous methods are appealing. $\mathrm{Re}$ expansion of an endograft with a large balloon, although effective in one of the patients in the series by Muhs and associates, ${ }^{8}$ still carries a risk of recurrence. In the same patient reported by Idu and coworkers ${ }^{6}$ and Hoornweg and colleagues, ${ }^{5}$ in one of the patients reported by Orend and coworkers, ${ }^{10}$ and in one of our patients, recurrence was directly observed under fluoroscopic or transesophageal echocardiographic imaging within seconds after deflation of the balloon. Placement of a reinforcing bare stent or stents confers some additional security but requires larger femoral arterial access. Furthermore, it is still no guarantee against recurrence because 1 patient in the series reported by Muhs and colleagues $^{8}$ suffered a recurrent collapse, even after the TAG had been re-expanded and reinforced with a Palmaz stent.

A previously unreported phenomenon seen in our series is the incompletely expanded device that was coaxed to expand spontaneously without further balloon dilatation or supplemental stenting. In this patient with a subacute dissection, false lumen depressurization and thrombosis was achieved by means of coil embolization of the covered LSCA and a large intercostal artery, allowing the true lumen to enlarge and to accommodate expansion of the TAG. All reinterventions in this patient were done through $5 \mathrm{~F}$ arterial sheaths and without general anesthesia. However, the final unfolding of the device apparently eroded through the dissection flap and formed an ulcer.

Our case of the delayed collapse of an original Thoracic Excluder is also unlike the other reported cases. Although retrospective analysis of the kinking of the aorta, the oversizing of the device, and the lack of apposition of the leading lip of the device help to explain the collapse, its occurrence more than 6 years after successful exclusion of a pseudoaneurysm is troubling. The device achieved its original goal of eliminating the aneurysm so well that the geometry of the aorta became less suitable for device integrity, resulting in a spine fracture and collapse. Unfortunately, this reinforces admonitions from skeptics who raise concerns about the durability of endograft repairs. As this technology is applied to younger patients with longer life expectancies, lifelong surveillance becomes a more challenging task.

\section{References}

1. Instructions for use for TAG thoracic endoprosthesis. Flagstaff, Ariz: W. L. Gore \& Associates, Inc; 2005.

2. Canaud L, Alric P, Branchereau P, Marty-Ane C, Berthet JP. Lessons learned from midterm follow-up of endovascular repair for traumatic rupture of the aortic isthmus. J Vasc Surg. 2008;47:733-8.

3. Go MR, Barbato JE, Dillavou ED, Gupta N, Rhee RY, Makaroun MS, et al. Thoracic endovascular aortic repair for traumatic aortic transection. J Vasc Surg. 2007;46:928-33

4. Hinchliffe RJ, Krasznai A, SchultzeKool L, Blankensteijn JD, Faleknberg M, Lonn L, et al. Observations on the failure of stent-grafts in the aortic arch. Eur J Endovasc Surg. 2007;34:451-6.

5. Hoornweg LL, Dinkelman MK, Goslings JC, Reekers JA, Verhagen HJM, Verhoeven EL, et al. Endovascular management of traumatic ruptures of the thoracic aorta: a retrospective multicenter analysis of 28 cases in The Netherlands. J Vasc Surg. 2006;43:1096-102.

6. Idu MM, Reekers JA, Balm R, Ponsen KJ, de Mol BAJM, Legemate DA. Collapse of a stent-graft following treatment of a traumatic thoracic aortic rupture J Endovasc Ther. 2005;12:503-7.

7. Melissano G, Tshomba Y, Civilini E, Chiesa R. Disappointing results with a new commercially available thoracic endograft. J Vasc Surg. 2004;39:124-30.

8. Muhs BE, Balm R, White GH, Verhagen HJM. Anatomic factors associated with acute endograft collapse after Gore TAG treatment of thoracic aortic dissection or traumatic rupture. J Vasc Surg. 2007;45:655-61.

9. Neschis DG, Moaine S, Gutta R, Charles K, Scalea TM, Flinn WR, et al. Twenty consecutive cases of endograft repair of traumatic aortic disruption: lessons learned. J Vasc Surg. 2007;45:487-92.

10. Orend KH, Zarbis N, Schelzig H, Halter G, Lang G, Sunder-Plassman L. Endovascular treatment (EVT) of acute traumatic lesions of the descending thoracic aorta-7 years' experience. Eur J Vasc Endovasc Surg. 2007;34:666-72.

11. Steinbauer MGM, Stehr A, Pfister K, Herold T, Zorger N, Topel I, et al. Endovascular repair of proximal endograft collapse after treatment for thoracic aortic disease. J Vasc Surg. 2006;43:609-12.

12. Tehrani HY, Peterson BG, Katariya K, Morasch MD, Stevens R, DiLuozzo G, et al. Endovascular repair of thoracic aortic tears. Ann Thorac Surg. 2006;82: 873-8.

13. Online calculator. Available at: http://ca.geocities.com/xpf51/MATHREF/ ELLIPSE.html. Accessed August 25, 2008.

14. Gavant ML. Helical CT grading of traumatic aortic injuries: impact on clinical guidelines for medical and surgical management. Radiol Clin North Am. 1999; 37:553-74.

15. Makaroun MS, Dillavou ED, Kee ST, Sicard G, Chaikof E, Bavaria J, et al Endovascular treatment of thoracic aortic aneurysms: results of the phase II multicenter trial of the Gore TAG thoracic endoprosthesis. J Vasc Surg. 2005;41:1-9.

16. Gore TAG thoracic endoprosthesis annual clinical update, April 2008. Available at: http://www.goremedical.com/en/file/AK0314.pdf. 